\title{
TWO STEP EXPLICIT FINITE ELEMENT METHOD FOR HIGH REYNOLDS NUMBER VISCOUS FLUID FLOW
}

\author{
By Mutsuto KAWAHARA* and Hirokazu HIRANO**
}

\section{INTRODUCTION}

In recent years, there have been presented a tremendous number of the finite element methods in the field of incompressible viscous fluid flow. One of the successful methods is referred to as the penalty function method, which has been investigated by Hughes et al.1),2), Heinlich et al. ${ }^{8)}, \operatorname{Reddy}^{4), 5)}$, Oden $^{6)}$ and Lee et al ${ }^{7)(9)}$. Hughes et al. have presented the method based on the reduced integration and solved steady flow at Reynolds number of $1.0 \times 10^{4}$. Lee and his group have investigated the penalty function method for both steady and unsteady flow. They have already presented the flow analysis at Reynolds number of $1.0 \times 10^{5}$. However, since their method is based on the implicit formulation, rather coarse finite element mesh has been used.

The basic idea of the penalty function method has already been found in the finite difference analysis, for instance, Chorin ${ }^{7}$, Marshall and Van Spiegel ${ }^{11)}$. Temam ${ }^{12), 13)}$ and Hirt and Nichols ${ }^{14}$. In those papers, the idea of the artificial compressibility can be found. Temam has also discussed the convergence of the finite element method using artificial compressibility. However, he has never shown the computational results.

This paper presents a finite element method for unsteady time dependent viscous incompressible fluid flow. As is described in the conventional finite element method, the equation of continuity for the incompressible flow is independent of time derivative of pressure. Because of this, it is impossible to use the explicit type of numerical integration in time. In addition, the diagonal terms of the coefficient matrix to be solved in the implicit scheme includes partly zero value, which extremely reduce the conditioning of the simultaneous equation systems. On the

* Member of JSCE, Department of Civil Engineering, Chuo University

** Member of JSCE, Mitsui Engineering and Shipbuilding, Co., Ltd. contrary, the physical material existing in the natural world have compressibility, i.e., the bounder value of the sound speed. Considering this fact, it is reasonable to transform the basic equation including the existing compressibility, which would be a large number, but, should be a limited amount. The formulation starting from the equations of state and conservation of mass leads to the equation including the terms of time derivative of pressure. If the sound speed tends to infinite, then the derived equation become coincident with the conventional equation of continuity. This formulation also corresponds to the physical interpretation of the penalty function method.

Contrary to the finite element methods previously published, the present method employs the explicit type of numerical integration in time. This is because the explicit scheme can save the computational core storage and time. The numerical test results obtained by the present method has been compared with the analytical solution and the numerical solution obtained by Lee et al. ${ }^{7)}$ Both tests illustrate the adaptability of the present finite element method.

Vortex shedding analysis has been performed using circular cylinder located in the air flow. The vortex shedding behind the cylinder has clearly been shown and Strouhal number has been compared with the experimental results. Both numerical and experimental values are well in agreement. The pressure coefficient around cylinder has also been compared with the experimental value. This paper will show that the present finite element method can be adaptable for the analysis of structures of arbitrary shape in the transient fluid flow subjected to fluid force such as vortex shedding.

\section{BASIC EQUATIONS}

Throughout this paper, equations are described by using indecial notation and summation convention with repeated indices. The analysis in this paper is restricted to two dimensional flow, however, the extension of the theory to the 
three dimensional problem is straight forward. The spatial rectangular coordinate and time are represented by $x_{i}$ and $t$ respectively. Notation ( ),i means partial differentiation with respect to $x_{i}$. Superposed $\cdot$ is differentiation with respect to $t$. The analysis presented in this paper aims to solve incompressible viscous flow introducing slight compressibility. The flow is assumed to be compressible, however, the compressibility is not so strong, then, the density of fluid flow $\rho$ can be taken as a constant with respect to $x_{i}$ and $t$.

Denoting fluid velocity by $v_{i}$, conservation of mass can be expressed as follows.

$$
\frac{\partial \rho}{\partial t}+\left(\rho v_{i}\right)_{, i}=0
$$

Using conservation of linear momentum, the equation of motion is written as:

$$
\rho\left(\frac{\partial v_{i}}{\partial t}+v_{\mathrm{J}} v_{i, \mathrm{j}}\right)+p, i-\tau_{i \mathrm{j}, \mathrm{j}}+\rho f_{i}=0 \cdots \cdots \cdots
$$

where $p$ and $f_{i}$ denote pressure and body force respectively. For the constitutive law of viscous stress $\tau_{i j}$, linear viscous fluid is assumed.

$$
\tau_{i j}=\kappa d_{k k} \delta_{i j}+2 \mu d_{i j}
$$

where $\kappa$ and $\mu$ mean volumatic and shear viscous coefficients. Kronecker delta function is represented by $\delta_{i j}$. Deformation rate $d_{i j}$ is derived from velocity:

$$
d_{i j}=\frac{1}{2}\left(v_{i, j}+v_{j, i}\right)
$$

Since the pressure is the function of density, Eq. of state for the temperature independent material can be written in the following form.

$$
p=p(\rho)
$$

Instead of introducing the explicit form of equation (5), the sound speed $c$ will be employed, i.e.,

$$
c^{2}=\frac{\partial p}{\partial \rho}
$$

Regarding boundary conditions, the following two types of condition will be considered. The first condition is the condition for velocity, i.e., the velocity is assumed to be prescribed on boundary $S_{1}$,

$$
v_{i}=\hat{v}_{i} \quad \text { on } S_{1}
$$

and the second condition is for the surface force, i.e., the surface force $S_{i}$ is given on boundary $S_{2}$,

$$
S_{i}=\left(-p \delta_{i j}+\tau_{i j}\right) n_{j}=\hat{S}_{i} \quad \text { on } S_{2}
$$

where superposed ${ }^{\wedge}$ denotes prescribed function on the boundary and $n_{j}$ is the unit normals to the boundary. The present paper considers that the whole boundary $S$ consists of only $S_{1}$ and $S_{2}$, and the following assumption is used.

$$
\left.\begin{array}{l}
S_{1} \cup S_{2}=S \\
S_{1} \cap S_{2}=\phi
\end{array}\right\}
$$

where $\phi$ is the null set. Eqs. (1) through (9) are taken as the basic equations of the analysis presented in this paper.

\section{TRANSFORMATION OF BASIC EQUATION}

The basic equations can be transformed into more convenient form for the finite element analysis. The material differentiation with respect to $t$ of Eq. (5) leads to the following equation.

$$
\frac{D p}{D t}=\frac{\partial p}{\partial \rho} \cdot \frac{D \rho}{D t}
$$

where $D / D t$ means material derivative. Introducing Eq. (1) into Eq. (10) and using (6), the equation of state can be reformulated as:

$$
\frac{\partial p}{\partial t}+v_{i} p, i+\rho c^{2} v_{i, i}=0
$$

The present method will employ Eq. (11) as the equation of continuity. If the sound speed $i$ tends to infinite, then Eq. (11) becomes the conventional form of equation of continuity as:

$$
v_{i, i}=0
$$

Almost all of the finite element methods previously published have used Eq. (12) as the equation of continuity. However, it is impossible that the explicit finite element method is formulated based on Eq. (12). This is the reason why the present paper prefers to use Eq. (11) as the equation of continuity. Eq. (11) also devotes the physical meaning of the so-called penalty function formulation.

Introducing Eqs. (3) and (4) into Eq. (2) and rearranging the terms, the equation of motion is derived as:

$$
\begin{gathered}
\rho\left(\frac{\partial v_{i}}{\partial t}+v j v_{i, j}\right)+p, i-\kappa v_{k, k_{i}} \\
-\mu\left(v_{i, j}+v_{j, i}\right), j+\rho f_{i}=0 \ldots
\end{gathered}
$$

In case of the analysis for the incompressible flow, the volumatic viscosity is taken to be zero, since $\kappa$ is introduced as the equation includes the compressibility. Taking the reference length and velocity as $L$ and $V$, the coordinate and time can be non-dimensionalized as:

$$
X_{i}=\frac{x_{i}}{L}, \quad T=\frac{t V}{L}
$$

Transforming Eqs. (11) and (13) into non-dimensional form, the equation of continuity and the equation of momentum can be expressed in the following forms.

$$
\frac{\partial P}{\partial T}+u_{i} P, i+C u_{i, i}=0
$$




$$
\begin{gathered}
\frac{\partial u_{i}}{\partial T}+u_{j} u_{i, j}+C P, i-\lambda u_{k, k_{i}} \\
-v\left(u_{i, j}+u_{j, i}\right), j+F_{i}=0
\end{gathered}
$$

where

$$
\begin{aligned}
& u_{i}=\frac{v_{i}}{V}, \quad P=\frac{p}{\rho c V}, \quad \lambda=\left(\frac{\kappa}{\rho}\right)\left(\frac{1}{V L}\right) \\
& \nu=\left(\frac{\mu}{\rho}\right) \cdot \frac{1}{V L}=\frac{1}{R e}, \quad C=\frac{c}{V}=\frac{1}{M c} \\
& F_{i}=\frac{L}{V^{2}} f_{i}
\end{aligned}
$$

in which $u_{i}, P$ and $C$ represent nondimensional velocity, pressure and velocity and $\lambda, \nu$ and $F_{i}$ are nondimensional viscosities and body force, and $R e$ and $M c$ are Reynolds number and Mach number respectively.

In almost similar manner, boundary conditions are reformulated as follows.

$$
\begin{aligned}
& u_{i}=\hat{u}_{i} \quad \text { on } S_{1} \\
& R_{i}=\left(-C P \delta_{i j}+t_{i j}\right) n_{j}=\hat{R}_{i} \quad \text { on } S_{2}
\end{aligned}
$$

where

$$
R_{i}=\frac{S_{i}}{\rho V^{2}}, \quad t_{i f}=\frac{\tau_{i j}}{\rho V^{2}}
$$

The finite element method presented in this paper is based on Eqs. (16) and (17) with boundary conditions (18) and (19).

\section{WEIGHTED RESIDUAL EQUATIONS}

Following the conventional Galerkin finite element procedure, the weighted residual equations and the interpolation equations are introduced. Let $P^{*}$ be the weighting function for pressure, the value of which is arbitrary. Multiplying both sides of Eq. (16) by $P^{*}$ and integrating over the volume $V$, the following equation is obtained.

$$
\begin{gathered}
\int_{V}\left(P^{*} \frac{\partial P}{\partial T}\right) d V+\int_{V}\left(P^{*} u_{i} P, i\right) d V \\
+\int_{V}\left(P^{*} C u_{i, i}\right) d V=0 \ldots \ldots \ldots \ldots . .
\end{gathered}
$$

Let $u_{i}{ }^{*}$ be the weighting function for velocity, the value of which is arbitrary except on boundary $S_{1}$ where it takes value zero. Multiplying both sides of Eq. (17) by $u_{i}^{*}$ and integrating over the volume $V$, then it follows that

$$
\begin{aligned}
& \int_{V}\left(u_{i} * \frac{\partial u_{i}}{\partial T}\right) d V+\int_{V}\left(u_{i} * u_{j} u_{i, j}\right) d V \\
& \quad+\int_{V}\left(u_{i} * C P, i\right) d V-\int_{V}\left(u_{i} * \lambda u_{k, k_{i}}\right) d V \\
& \quad-\int_{V} u_{i} * v\left(u_{i, j}+u_{j, i}\right), j d V+\int_{V}\left(u_{i} * F_{i}\right) d V=0
\end{aligned}
$$

Integrating the third, fourth and fifth terms of Eq. (21) by parts, the weighted residua lequation can be derived in the following form.

$$
\begin{aligned}
& \int_{V}\left(u_{i} * \frac{\partial u_{i}}{\partial T}\right) d V+\int_{V}\left(u_{i} * u_{j} u_{i, j}\right) d V \\
& \quad-\int_{V}\left(u_{i, i}^{*} C P\right) d V+\lambda \int_{V}\left(u_{i, i}^{*} u_{k, k}\right) d V \\
& \quad+\nu \int_{V}\left(u_{i, j}^{*} u_{i, j}\right) d V+\nu \int_{V}\left(u_{i, j}^{*} u_{j, i}\right) d V \\
& \quad+\int_{V}\left(u_{i}^{*} F_{i}\right) d V=\int_{S_{2}}\left(u_{i} * \hat{R}_{i}\right) d S \ldots .
\end{aligned}
$$

Eq. (22) includes the boundary condition of Eq. (19) on $S_{1}$ as the natural boundary condition. Therefore, the weighted residual Eqs. (20) and (22) with boundary condition (18) can be adaptable for the finite element analysis.

\section{FINITE ELEMENT ANALYSIS}

Assume that the flow field to be analyzed is divided into a number of small domains called finite elements. For velocity, the interpolation equation is introduced as:

$$
\begin{aligned}
& u_{i}=\Phi_{\alpha} u_{\alpha i} \\
& u_{i}{ }^{*}=\Phi_{\alpha} u_{\alpha i}^{*}
\end{aligned}
$$

where $\Phi_{\alpha}$ is the interpolation function, $u_{\alpha i}$ denotes nodal value of velocity at $\alpha$ th node of the finite element in the $i$ th direction and $u_{\alpha i}^{*}$ represents the corresponding nodal value of weighting function respectively. For pressure, the following equation is used.

$$
\begin{aligned}
& P=\Phi_{\alpha} P_{\alpha} \\
& P^{*}=\Phi_{\alpha} P_{\alpha}{ }^{*}
\end{aligned}
$$

where $P_{\alpha}$ and $P_{\alpha} *$ denote nodal value of pressure at $\alpha$ th node of the finite element and the corresponding nodal value of weighting function respectively. The interpolation function is essentially related to the stability of a numerical integration scheme in time. Considering that the present method employs the two step explicit scheme for the numerical integration in time, the linear interpolation function based on three node triangular finite element is used for both velocity and pressure.

Substituting Eqs. (23) through (26) into Eqs. (20) and (22), and considering the arbitrariness of $u_{\alpha i}^{*}$ and $P_{\alpha}{ }^{*}$, the finite element equation can be derived as follows.

$$
\begin{aligned}
& M_{\alpha \beta} \dot{u}_{\beta i}+K_{\alpha \beta \gamma} j u_{\beta j} u_{\gamma i}-C H_{\alpha i \beta} P_{\beta}+S_{\alpha i \beta j u_{\beta j}} \\
& =\hat{\Omega}_{\alpha i} \\
& M_{\alpha \beta} \dot{P}_{\beta}+A_{\alpha \beta i} P_{\beta} u_{\gamma i}+C B_{\alpha \beta i} u_{\beta i}=0
\end{aligned}
$$

where

$$
M_{\alpha \beta}=\int_{V}\left(\Phi_{\alpha} \Phi_{\beta}\right) d V
$$




$$
\begin{aligned}
K_{\alpha \beta \gamma}= & \int_{V}\left(\Phi_{\alpha} \Phi_{\beta} \Phi_{\gamma, j}\right) d V \\
H_{\alpha i \beta}= & \int_{V}\left(\Phi_{\alpha, i} \Phi_{\beta}\right) d V \\
S_{\alpha i \beta j}= & \lambda \int_{V}\left(\Phi_{\alpha, i} \Phi_{\beta, j}\right) d V+\nu \int_{V}\left(\Phi_{\alpha, i} \Phi_{\beta, j}\right) d V \\
& +\nu \int_{V}\left(\Phi_{\alpha, k} \Phi_{\beta, k}\right) \delta_{i j} d V \\
\hat{\Omega}_{\alpha i}= & \int_{S_{2}}\left(\Phi_{\alpha} \hat{R}_{i}\right) d S-\int_{V}\left(\Phi_{\alpha} F_{i}\right) d V \\
A_{\alpha \beta i \gamma}= & \int_{V}\left(\Phi_{\alpha} \Phi_{\beta, i} \Phi_{\gamma}\right) d V \\
B_{\alpha \beta i}= & \int_{V}\left(\Phi_{\alpha} \Phi_{\beta, i}\right) d V
\end{aligned}
$$

For the numerical integration in time, the selective lumping two step explicit scheme is introduced. The scheme has already been investigated by Kawahara et al. ${ }^{15)} 22$ ) in case of the shallow water equations. The scheme consists of two steps and can be expressed as follows For the first step:

$$
\begin{aligned}
& \bar{M}_{\alpha \beta} u_{\beta i}^{n+1 / 2}=\bar{M}_{\alpha \beta} u_{\beta i}^{n}-\frac{\Delta t}{2}\left(K_{\alpha \beta \gamma} j u_{\beta j}^{n} u_{r i}^{n}\right. \\
& \left.-C H_{\alpha i \beta} P_{\beta}{ }^{n}+S_{\alpha i \beta j} u_{\beta j}^{n}-\hat{\Omega}_{\alpha i}^{n}\right) \\
& \bar{M}_{\alpha \beta} P_{\beta i}^{n+1 / 2}=\tilde{M}_{\alpha \beta} P_{\beta}{ }^{n}-\frac{\Delta t}{2}\left(C B_{\alpha \beta i} u_{\beta i}^{n}\right. \\
& \left.+A_{\alpha \beta i \gamma} P_{T}{ }^{n} u_{B i}^{n}\right)
\end{aligned}
$$

and for the second step;

$$
\begin{aligned}
\bar{M}_{\alpha \beta} u_{\beta i}^{n+1}= & \bar{M}_{\alpha \beta} u_{\beta i}^{n}-\Delta t\left(K_{\alpha \beta \gamma j} u_{\beta j}^{n+1 / 2} u_{\gamma i}^{n+1 / 2}\right. \\
& \left.-C H_{\alpha i \beta} P_{\beta}^{n+1 / 2}+S_{\alpha i \beta j} u_{\beta j}^{n}-\hat{\Omega}_{\alpha i}^{n}\right) \\
& \cdots \cdots \cdots \cdots \cdots \cdots \cdots \cdots \cdots \cdots \cdots \cdots \cdots \cdots \cdots \cdots \cdots \cdots \cdots \cdots \cdots \cdots \\
\bar{M}_{\alpha \beta} P_{\beta}^{n+1}= & \tilde{M}_{\alpha \beta} P_{\beta}{ }^{n}-\Delta t\left(C B_{\alpha \beta i} u_{\beta i}^{n+1 / 2}\right. \\
& \left.+A_{\alpha \beta i \gamma} P_{r i}^{n+1 / 2} u_{\beta i}^{n+1 / 2}\right)
\end{aligned}
$$

where $\bar{M}_{\alpha \beta}$ denotes the lumped coefficient and

$$
\tilde{M}_{\alpha \beta}=e \bar{M}_{\alpha \beta}+(1-e) M_{\alpha \beta} \text {. }
$$

in which $e$ is referred to as the selective lumping parameter, that is used to reduce the numerical damping effect and to adjust the numerical stability. How to choose the selective lumping parameter $e$ is precisely investigated by Kawahara et al. ${ }^{22}$ )

\section{NUMERICAL TEST EXAMPLE}

The first example is on propagation of pressure difference. This example will test the adaptability of the present finite element method, especially the choice of the selective lumping parameter. Consider one dimensional pressure difference propagation problem:

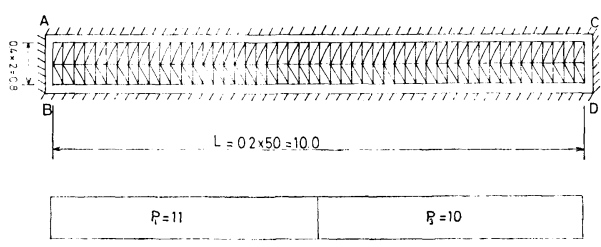

Fig. 1

$$
\begin{aligned}
& \frac{\partial u}{\partial T}+u \frac{\partial u}{\partial X}+C \frac{\partial P}{\partial X}=0 \\
& \frac{\partial P}{\partial T}+u \frac{\partial P}{\partial X}+C \frac{\partial u}{\partial X}=0
\end{aligned}
$$

where $u, P$ represent velocity and pressure and $\rho, C$ are density and sound speed respectively, both of which are assumed constant. The problems is wholly one dimensional, however, the two dimensional method of solution has been used based on the finite element idealization shown in Fig. 1 for the purpose to test the present finite element method. For the boundary condition, normal velocity on all boundaries is assumed to be zero. For the initial condition, the pressure difference between two regions are enforced as $P_{1}=11$ and $P_{3}=10$.

From Eqs. (34) and (35), the following equations can be derived.

$$
\begin{aligned}
& {\left[\frac{\partial}{\partial T}+(u+C) \frac{\partial}{\partial X}\right](u+P)=0} \\
& {\left[\frac{\partial}{\partial T}+(u-C) \frac{\partial}{\partial X}\right](u-P)=0}
\end{aligned}
$$

Therefore, $(u+P)$ and $(u-P)$ are conserved. At wave front, the conservation equations

$$
\begin{aligned}
& \lfloor\rho u \rrbracket=0 \\
& \backslash P+u / C \backslash=0
\end{aligned}
$$

are valid, where 【】denotes the value of difference at the wave front. Using the above equations, propagation wave can be expressed in Fig. 2. The propagation speed is $C$ and pressure in the intermediate region $P_{2}$ is obtained as follows.

$$
P_{2}=1 / 2\left(P_{1}+P_{3}\right)
$$

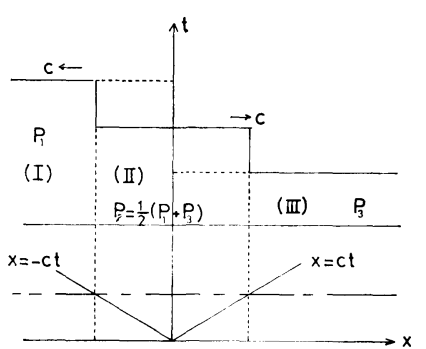

Fig. 2 


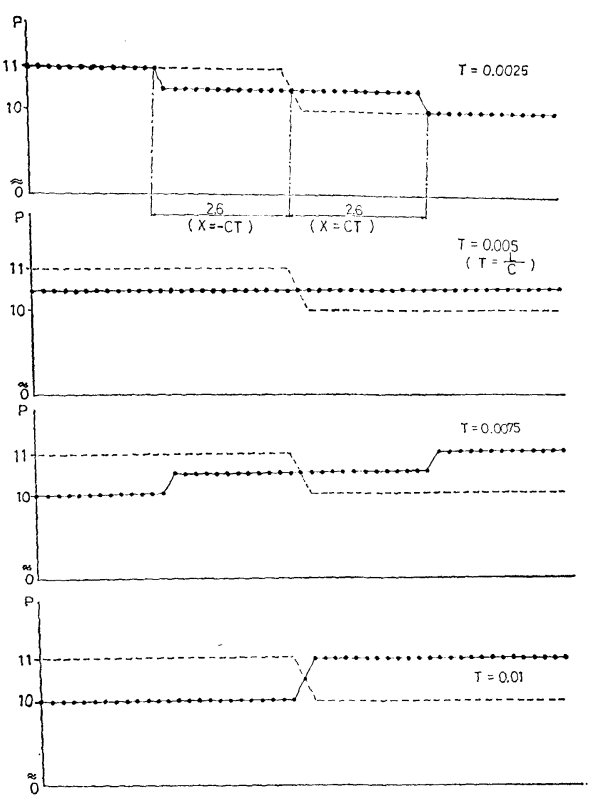

Fig. 3

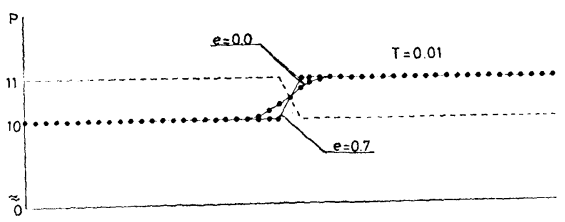

Fig. 4

Fig. 3 illustrates the computed wave propagation of pressure using the selective lumping parameter $e=0.7$ and $C=1000$. The wave propagation and the reflection of wave can be obtained exactly same as of analytical solution. The propagation time of the wave is also coincident with analytical solution.

Fig. 4 represents the reflected wave computed with the two kinds of selective lumping parameter. In case of $e=0$, the slight numerical damping effect is observed. From Figs. 3 and 4, it can be concluded that the wave propagation can be exactly computed using the proper choice of the selective lumping parameter. If the choice of the parameter is not suitable, the instability of the computation or the superfluous numerical damping will arise.

The second example is the computation of the flow through a channel with sudden expansion. The results have been compared with those obtained by R. L. Lee et al. ${ }^{7)}$ The finite element idealization and boundary conditions are shown in Fig. 5. At the side walls of the channel, both components of the velocity are assumed to be zero. At the entrance of the channel $(\mathrm{A} \sim \mathrm{E})$,
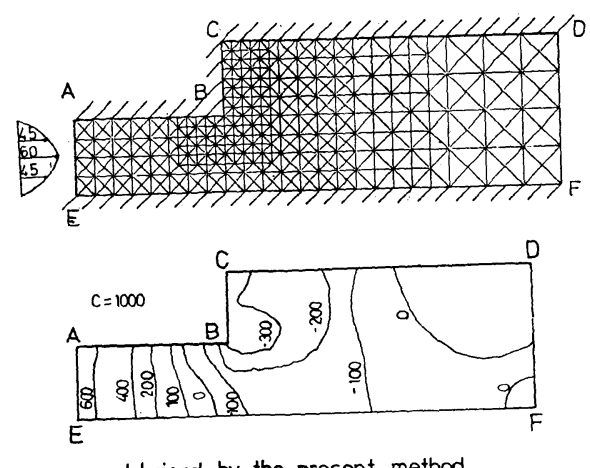

obtained by the present method
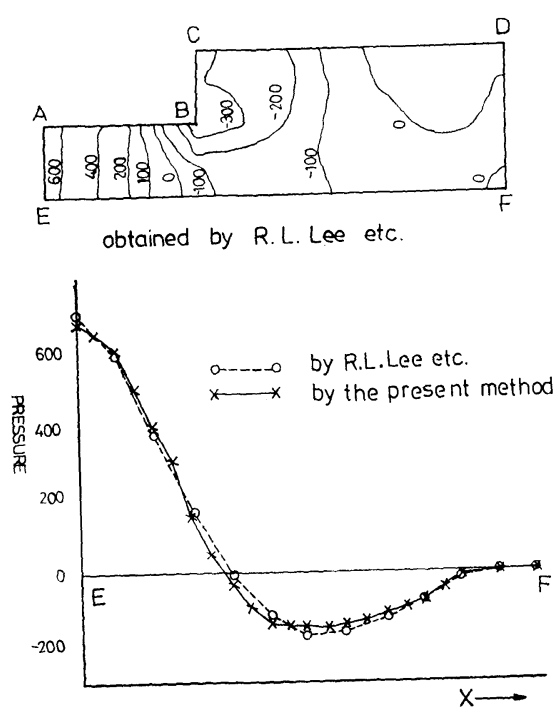

Fig. 5

the parabolic velocity profile is enforced. At the boundary $D \sim F$, surface force is given to be zero. The nondimensional sound speed $C=1000$ and the Reynolds number $R e=60$ are introduced where Reynolds number is computed by the maximum entrance velocity and entrance length. The computed pressure compared with the results by $R$. L. Lee et al. ${ }^{\text {7) }}$ is represented in the figure. The computed results by $R$. L. Lee et al. are obtained by the steady flow analysis based on the penalty function formulation. Both results are extremely well in agreement. It can be concluded from the figure that the present method is adaptable for the analysis of incompressible viscous fluid flow.

\section{VORTEX SHEDDING ANALYSIS}

The present finite element method has been applied to the analysis of vortex shedding behind cylinder. Fig. 6 shows the finite element idealization used in the analysis. Total numbers of nodal 


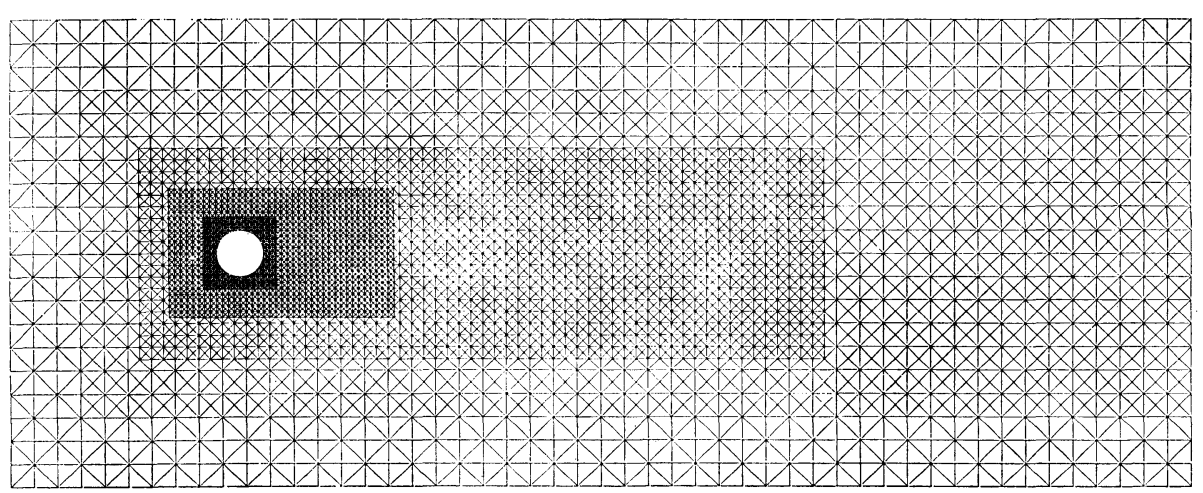

Fig. 6

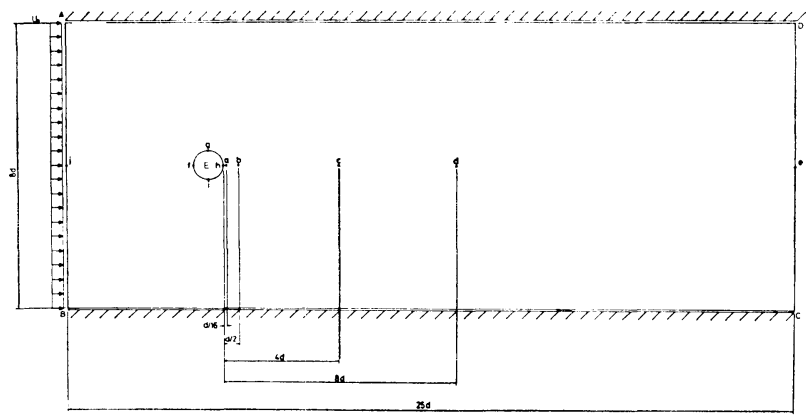

Fig. 7

points and finite elements are 4764 and 9340 respectively. The diameter of the cylinder is denoted by $d$. The distance between the entrance of the channel and leftest end of the cylinder is $4.4 \mathrm{~d}$. The channel width $\mathrm{A}-\mathrm{B}$ is employed to be $8 \mathrm{~d}$. From the various numerical experiences, it is seen that the channel width affects the computed velocity if less than $8 \mathrm{~d}$ channel width is used.

To examine the effect of the location of arbitrary boundary $\mathrm{C}-\mathrm{D}$, the computation using the longer channel length ( $36 \mathrm{~d}$ ) has been performed. For the computed results during the first 1000 steps, there has been observed no significant discrepancy except pressure. For pressure, sinusoidal variation in time is computed, which corresponds to the sound wave travelling through the channel from $\mathrm{A}-\mathrm{B}$ to $\mathrm{D}-\mathrm{C}$ and its reflective wave. The period of this sinusoidal variation of pressure is related to the length of the channel. However, the pressure from which the pressure at the entrance of the channel is subtracted is not affected by the location of the artificial boundary C-D. Therefore, the computation has been continued using the channel length $25 \mathrm{~d}$ shown in Fig. 6.

Fig. 7 illustrates the boundary conditions and referring points, numerical results at which are shown in the following figures. On the boundaries $\mathrm{A}-\mathrm{D}$ and $\mathrm{B}-\mathrm{C}$, normal velocities to the boundaries and the tangential surface force are assumed zero. On the boundary D-C, both surface forces are specified to be zero. Uniform velocity profile with absolute value $U_{0}$ is enforced on boundary A-B. Both components of velocity on the cylinder surface are taken to be zero.

Referring to air flow, sound speed and density are $C=337$ and $\rho=0.1319 \mathrm{~kg} / \mathrm{m}^{3}$. For viscosity, $\nu=0.01$ and $\lambda=0.0$ are assumed. The selection of time increment $\Delta T$ depends on the following empirical condition,

$$
\Delta T \leqq \frac{\alpha \Delta x}{U_{0}+C}
$$

where $\alpha$ is an empirical constant, the value of which is $0.01 \sim 0.5$. Starting from zero initial condition, i.e., all velocity and pressure are given to be zero, the computation has been carried out using the time increment $\Delta T=2 \times 10^{-4}$ and the selective lumping parameter $e=0.73$. Reynolds number estimated is as

$$
R e=\frac{U_{0} d}{\nu}=1.5 \times 10^{5}
$$

where $U_{0}$ represents the entrance velocity.

Fig. 8 shows the computed velocity at time $T$ $=8.00$ through $T=8.12$. In this figure, vortex 


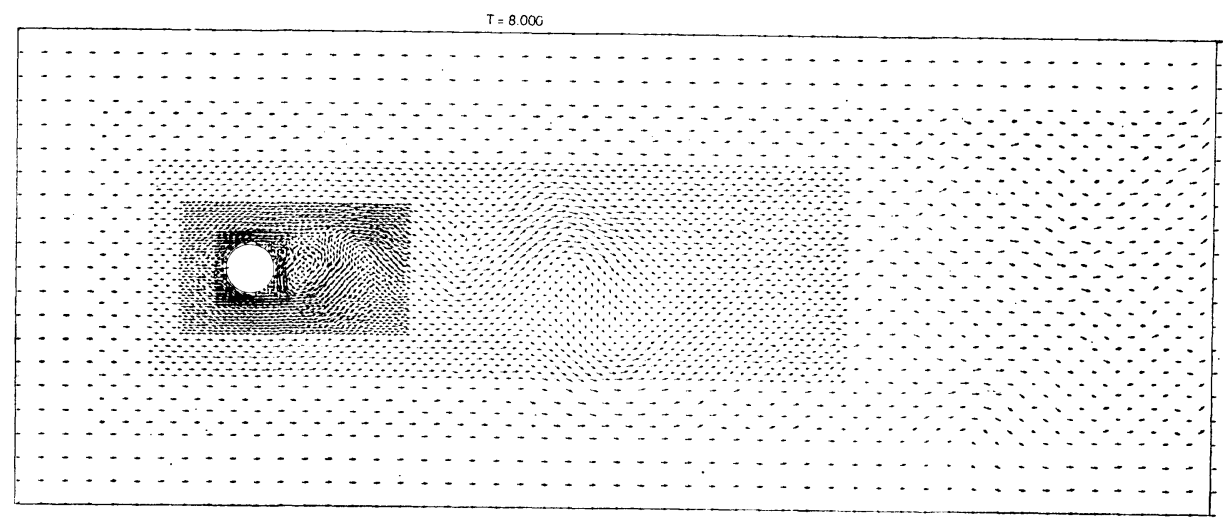

$T=8.040$

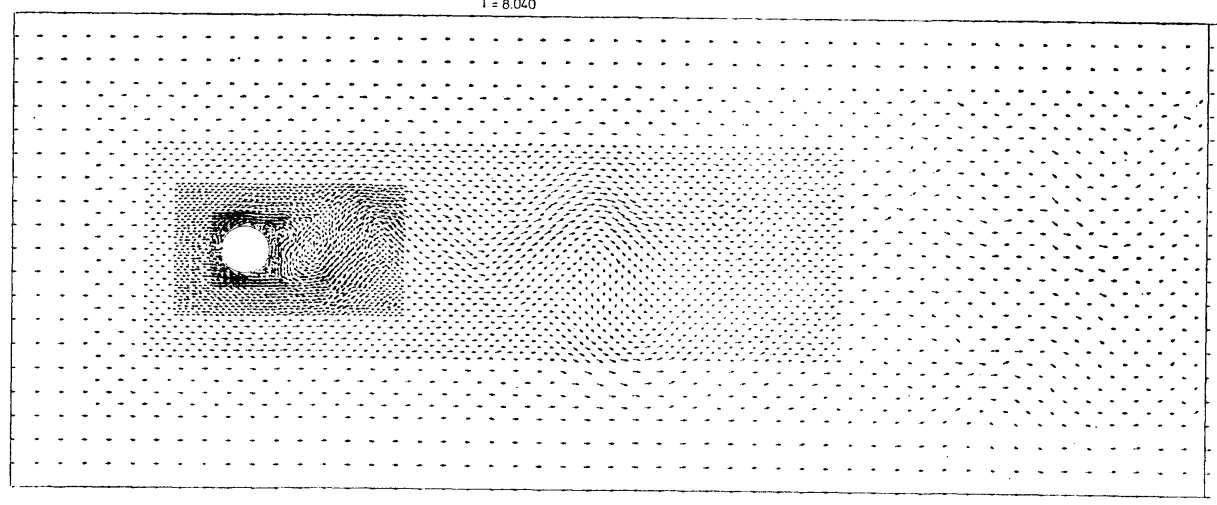

$T=8.080$

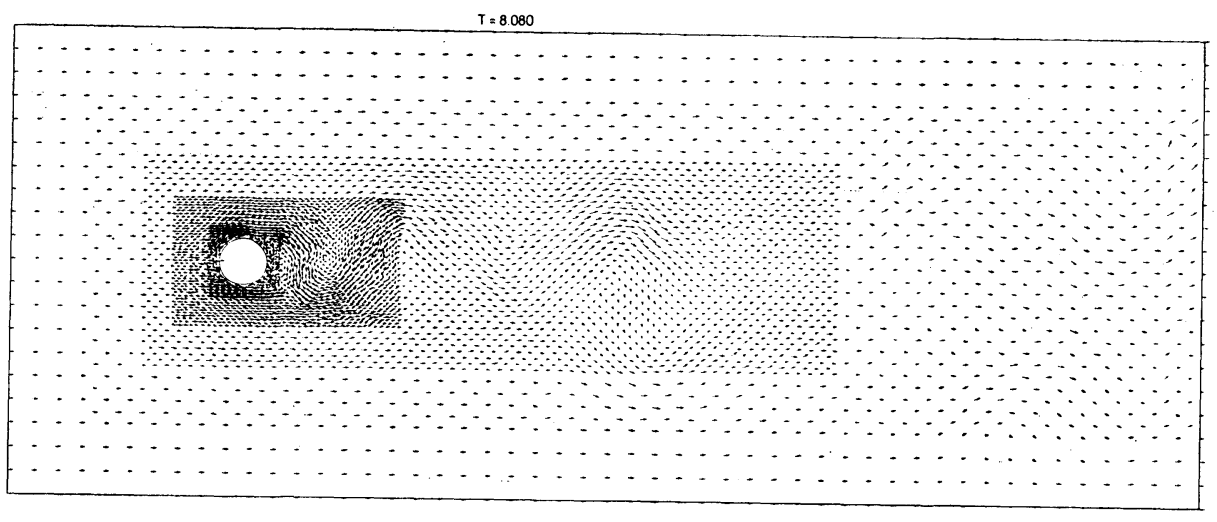

$T=8.120$

Fig. 8 

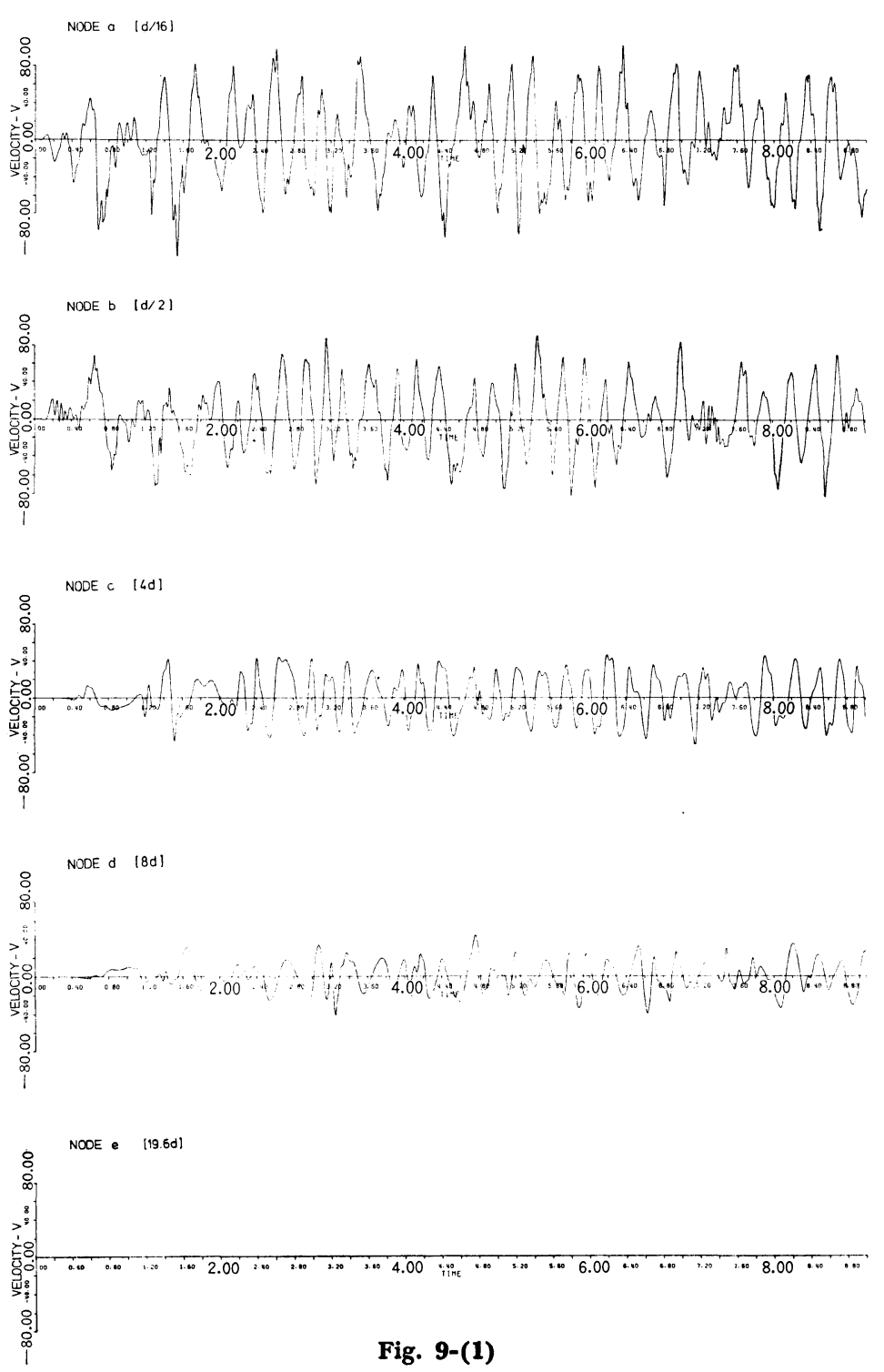

shedding can be clearly shown. The horizontal distance between vortices $b$ is computed from the results as $b=3.40 \mathrm{~d}$. The vertical distance between vortices is $a=0.85 \mathrm{~d}$. The ration $a / b$ is:

$$
a / b=0.25
$$

The theoretical value is 0.28 , which is reasonably well in agreement with that obtained by the computation. Using the convective velocity of vortex, the Strouhal number is computed as

$$
\begin{aligned}
& S=0.1875 \\
& 1 / S=5.33
\end{aligned}
$$

The experimental value of $1 / S$ is around 5.5 (e.g., Ito, Miyata and Okauchi ${ }^{28)}$ ), which is coincident with one obtained by the computation.
Fig. 9 shows the diagram of both components of velocity versus time at points $a, b, c, d$ and $e$. Horizontal component is denoted by $U$ and vertical component by $V$. The locations of the referring points are apart from the rightest end of the cylinder by $\mathrm{d} / 16, \mathrm{~d} / 2,4 \mathrm{~d}, 8 \mathrm{~d}$ and $19.6 \mathrm{~d}$, respectively. From the figure, it is seen that the vertical velocity components have larger values near the cylinder than those far from the cylinder. At the outlet of the channel, i.e., point e, vertical component is computed to be zero. At the points near the cylinder, e.g. points a and b, it is clearly shown that the frequency of the variation of horizontal velocity is as twice as that of vertical velocity. At the points slightly far from the points, e.g. points $\mathrm{c}$ and $\mathrm{d}$, the frequency of horizontal 

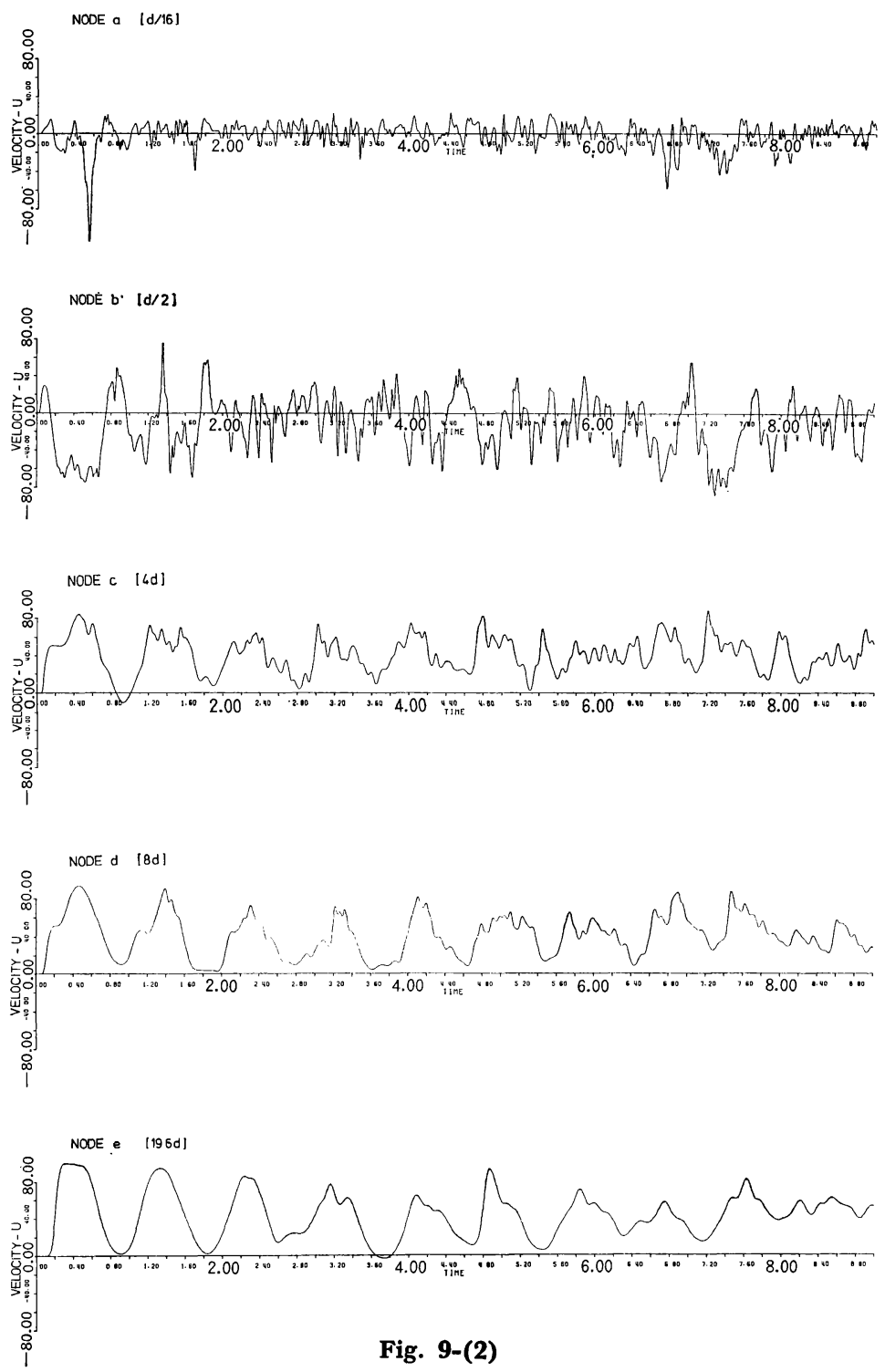

velocity tends to be similar to that of vertical velocity. These tendencies are very much alike to those of experimental results.

Fig. 10 represents the computed pressure $P$ at time $T=8.00$ through $T=8.12$. In the figure, transition of pressure distribution can be clearly shown. Locations and centers of vortices can be made clear distinction. Fig. 11 is the illustration of the diagram of the computed pressure at points e, $f, g, h, i$ and $j$, which are shown in Fig. 7. In the figure, pressure variation can be seen as a decaying sinusoidal function, of which period is about 1.8 nondimensional time. However, this sinusoidal variation should be considered as the sound wave which travels through the channel from $\mathrm{A}-\mathrm{B}$ to $\mathrm{D}-\mathrm{C}$. For the purpose of obtaining the information about the fluid force subject to the cylinder, it is necessary to eliminate the effect of this sinusoidal variation of sound wave. To do this, the following pressure coefficient $C_{p}$ has been computed.

$$
C_{p}=\frac{P-P_{0}}{(1 / 2) \rho U_{0}^{2}}
$$

where $P_{0}$ is the pressure computed at the center of the entrance channel, i.e., point $j$. The computed pressure coefficients at the surface of the cylinder, i.e., points $f, g, h$ and $i$, are plotted in Fig. 12. The phase lag of the variation of $C_{p}$ between at top of the cylinder, point $g$ and at 

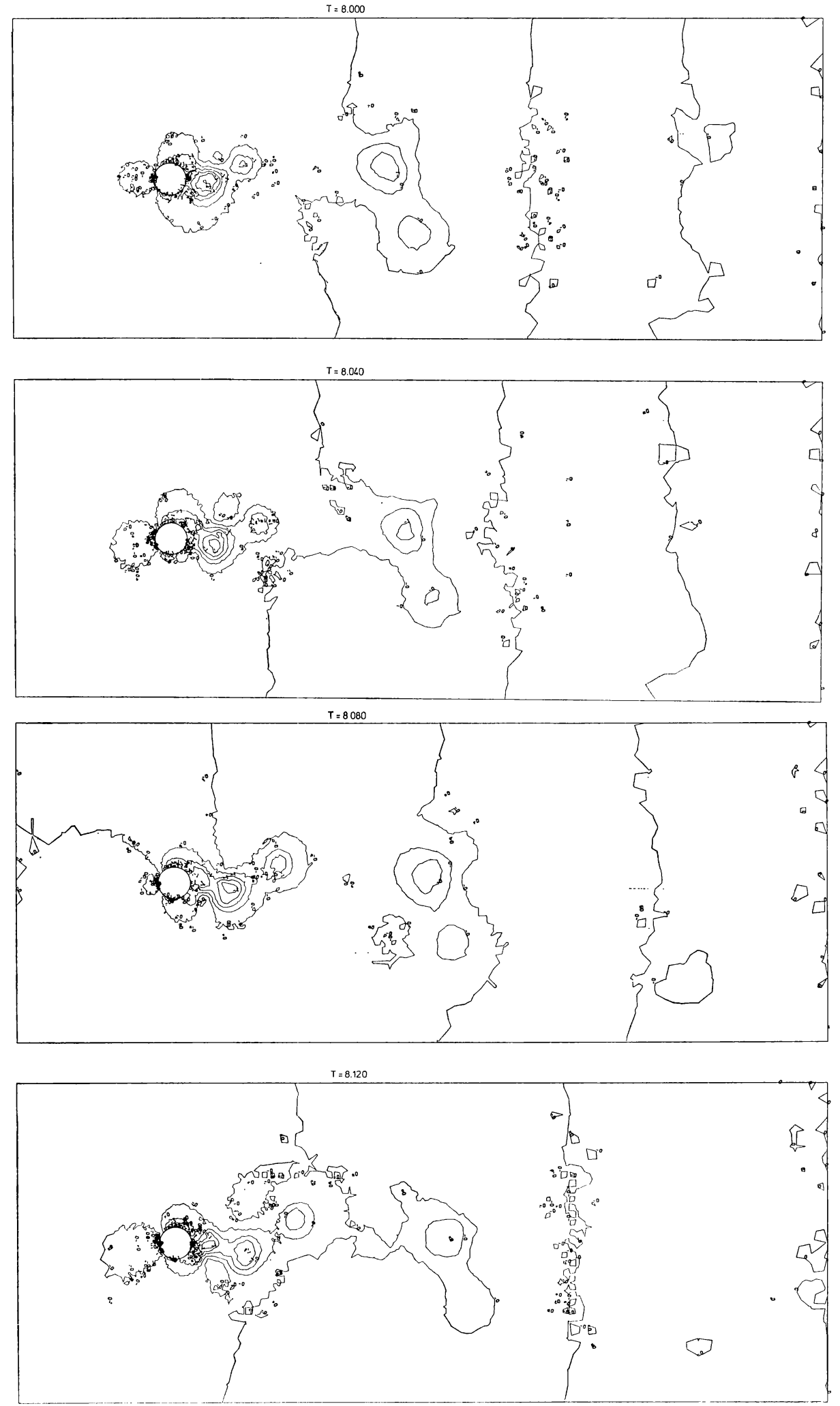

Fig. 10 


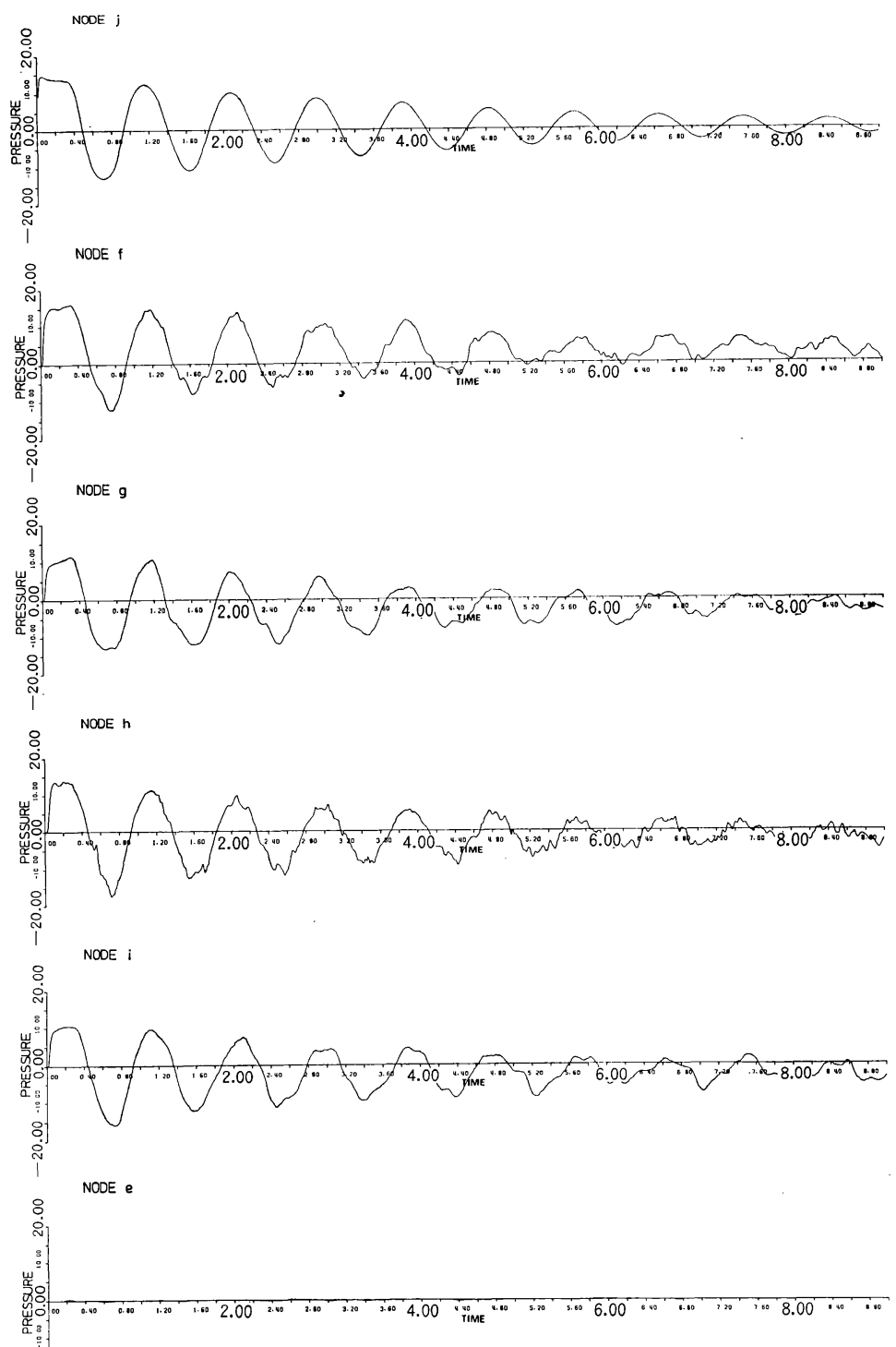

Fig. 11

bottom, point $\mathrm{i}$ is computed as $\Pi$. Moreover, the frequency of $C_{p}$ computed at points near end of the cylinder, point $h$, is as twice as those at top and bottom points, i.e., points $g$ and $i$. These tendency completely explains the vortex shedding behind cylinder and corresponds to the experimental results.

The drag and lift coefficients defined as

$$
\begin{aligned}
& C_{D}=\frac{D}{(1 / 2) \rho U_{0}^{2} d} \\
& C_{l}=\frac{L}{(1 / 2) \rho U_{0}^{2} d}
\end{aligned}
$$

are computed where $D$ and $L$ are horizontal and vertical, total surface forces calculated at the surface of the cylinder. The time variation is plotted in Fig. 13. The average values of both coefficients through total time interval are $\boldsymbol{C}_{\boldsymbol{D}}=$ 1.23 and $C_{l}=0.0$, respectively. These computed values are extremely well in agreement with the experimental data.

From the computed time variation of $C_{l}$, the frequency of the alternating force can be computed as the frequency of $C_{p}$ as follows.

$$
f^{\prime}=3.44
$$

Using this, the Strouhal number can be computed as

$$
S^{\prime}=\frac{f^{\prime} d}{U_{0}}=0.2064
$$

Therefore

$$
1 / S^{\prime}=4.85
$$



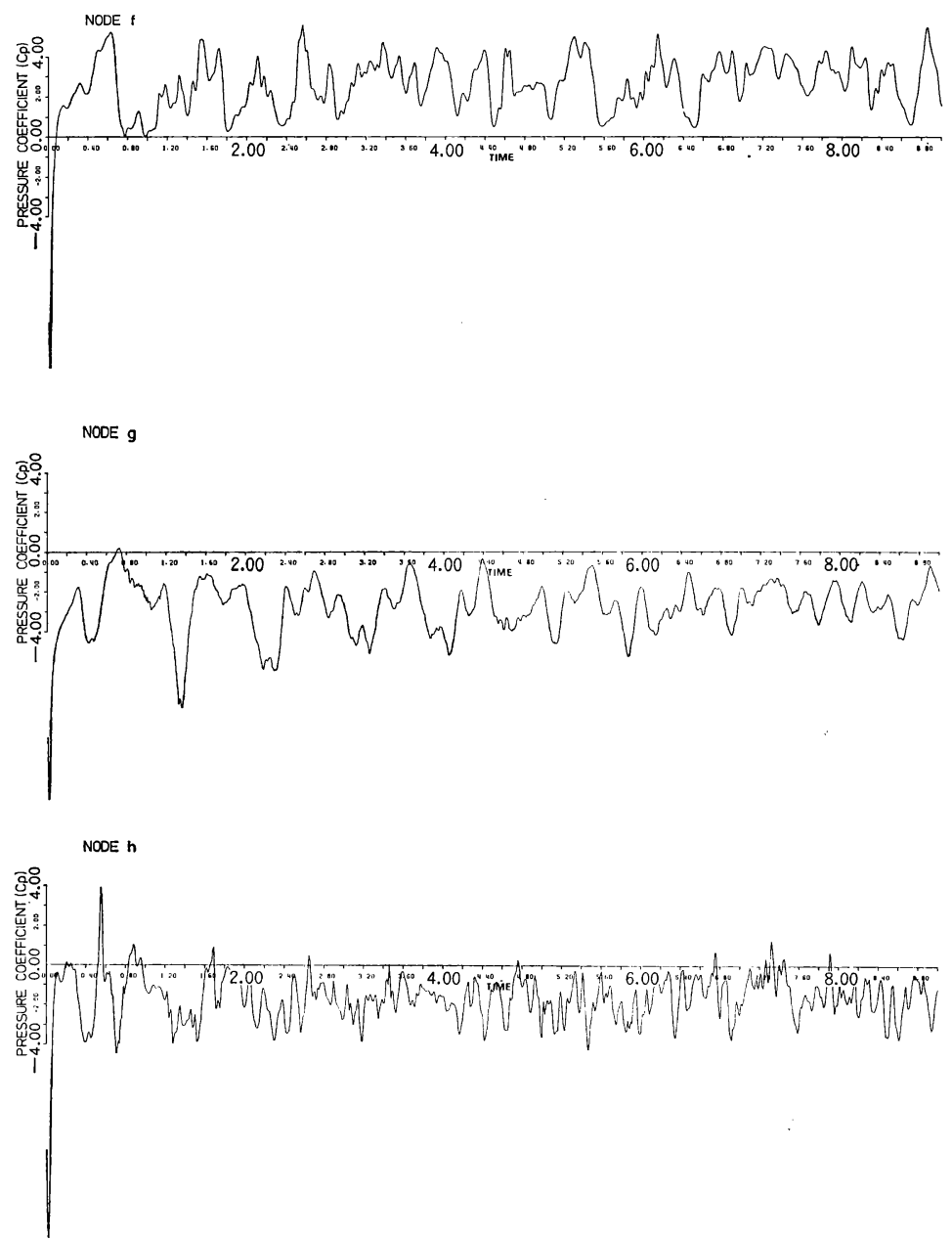

NOOE i

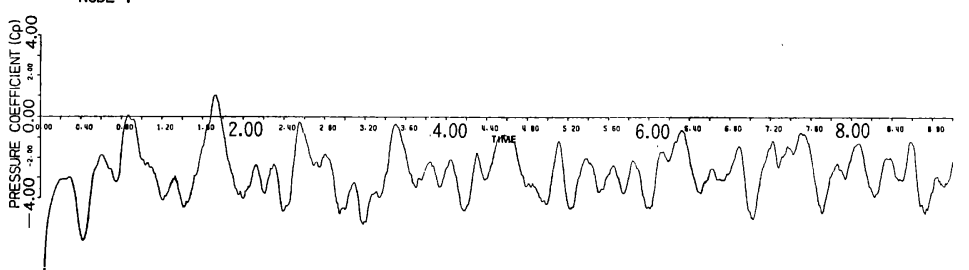

Fig. 12

which are reasonably well in agreement with the experimental results (e.g. Ito, Miyata and Okauchi ${ }^{23)}$ ).

In Fig. 14, average pressure coefficient around the surface of the cylinder is illustrated with the experimental data (from Ito, Miyata and Okauchi $\left.{ }^{23)}\right)$. Without few exceptions around singular points, both results show good agreement.

From these considerations, it can be stated that the numerical results obtained by the present finite element method explain exactly the behavior of vortex shedding behind cylinder. The present computation has been carried out using HITAC M280H. The CPU time was about 1 hour per each total 10000 steps.

\section{CONCLUSION}

In the present paper, a finite element method for unsteady two dimensional flow of incompressible viscous fluid at relatively high Reynolds 


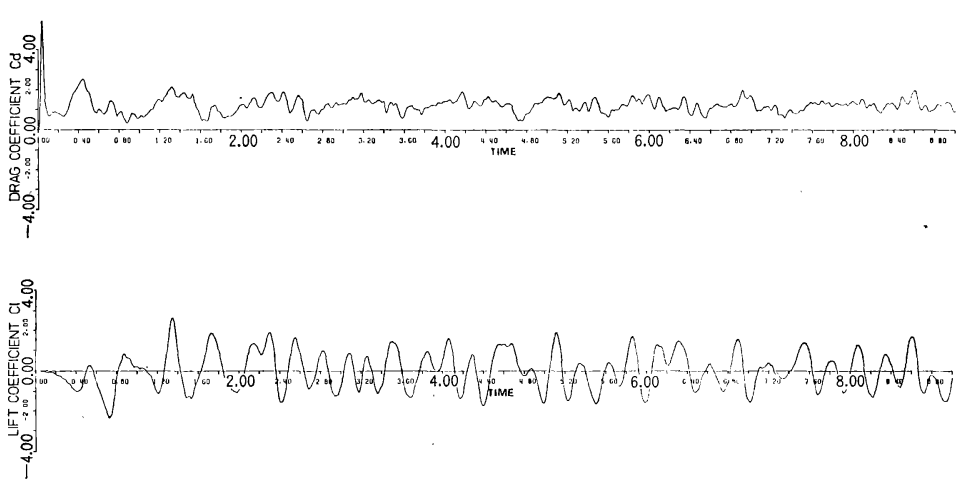

Fig. 13

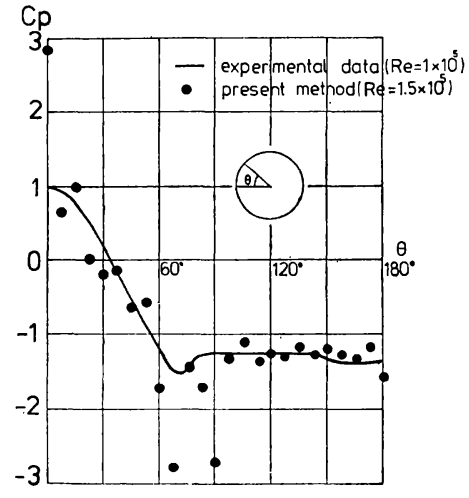

Fig. 14 number has been presented and applied to vortex shedding analysis. The finite element method is characterized by the following items.

(1) The method is based on the formulation including compressibility. Using the sound speed, the equations of state and conservation of mass can be transformed into the suitable form to the explicit numerical integration in time. The formulation corresponds to the physical interpretation of the penalty function method.

(2) The two step explicit numerical integration in time is used based on the linear triangular finite element. Using the explicit scheme, the core storage requirement and computational time are extremely reducible.

(3) The numerical results obtained by the present method has been compared with the results computed by the analytical method and the conventional numerical method. The results are extremely well in agreement.

(4) The flow passed cylinder has been computed at Reynolds number of $1.5 \times 10^{5}$. The vortex shedding has been clearly shown. Flow patterns, pressure distributions, Strouhal number and drag and lift coefficients are all well in agreement with those of experimental data.

(5) It is concluded that the finite element method presented in this paper can be adaptable for the analysis of fluid velocity and pressure distribution through channels, fluid machines, etc. of arbitrary configuration and of fluid force to structures, e.g. drag and lift forces, pressures. etc. The possibilities of application of the present finite element method is widely spread in all directions in the practical engineering fields.

To compute the flow at relatively high Reynolds number, for instance, vortex shedding behind cylinder, refined finite element idealization and short time increment usage are indispensable. For these reasons, the explicit numerical integration scheme is preferable to save the computa- tional core storage and time. For the explicit numerical integration in time, the selective lumping two step scheme using linear interpolation function is suitable considering numerical stability.

\section{ACKNOWLEDGEMENT}

Computations in this research have been carried out by Mr. Toshio Kodama, Graduate Student of Chuo University using FACOM 230$48 \mathrm{~S}$ of Chuo University and HITAC M280H of University of Tokyo. A part of this research has been supported by Grants-in-Aid for Scientific Research, No. 56550319, Ministry of Education, Government of Japan.

\section{REFERENCES}

1) Hughes, T. J. R., W. K. Liu and A. Brooks: Finite Element Analysis of Incompressible Viscous Flows by the Penalty Function Formulation, J. Computational Phys., Vol. 30, pp. 1 60, 1979.

2) Hughes, T. J. R., R. L. Taylor and J. Levy: High Reynolds Number, Steady, Incompressible Flows by a Finite Element Method, Finite Elements in Fluids, Vol. 3, (eds. by Gallagher et al.), John Wiley \& Sons, 1978.

3) Heinrich, J. C., R. S. Marshall and O. C. Zienkiewicz: Penalty Function Solution of Coupled Convective and Conductive Heat Transfer, Numerical Methods in Laminar and Turbulent Flow, eds. by C. Taylor et al., Pentech Press, pp. 935 946, 1978.

4) Reddy, J. N.: On the Finite Element Method with Penalty for Incompressible Fluid Flow Problems, The Mathematics of Finite Elements and Applications III, ed. by J. R. Whiteman, Academic Press, pp. 227 239, 1979.

5) Reddy, J. N.: On the Mathematical Theory of Penalty-Finite Elements for Navier-Stokes 
Equations, Proceedings of the Third International Conference on Finite Element Method in Flow Problems, pp. 146 154, the University of Calgary, 1980.

6) Oden, J. T.: Penalty Methods and Selective Reduced Integration for Stokesian Flows, Proceedings of the Third International Conference on Finite Element Method in Flow Problems, pp. $140 \sim 154$, the University of Calgary, 1980.

7) Lee, R. L., P. M. Gresho and R. L. Sani: Smoothing Techniques for Certain Primitive Variable Solutions of the Navier-Stokes Equations, Int. J. Num. Meth. Engng., Vol. 14, No. 12, pp. 1785 1804, 1979.

8) Lee, R. L., P. M. Gresho, S. T. Chan and R. L. Sani: A Comparison of Several Conservative Forms for Finite Element Formulations of the Incompressible Navier-Stokes or Boussinesq Equations Proceedings of the Third International Conference on Finite Element Method in Flow Problems, Banff, the University of Calgary, pp. 236 227, 1980.

9) Gresho, P. M., R. L. Lee and C. D. Upson: FEM Solution of the Navier-Stokes Equations for Vortex Shedding Behind A Cylinder: Experiments with the Four Node Element, Finite Elements in Water Resources, eds. by S. Y. Wang et al., the University of Mississippi, pp. 4.48 4.65, 1980.

10) Chorin, A. J.: A Numerical Method for Solving Incompressible Viscous Flow Problems, J. Computational Phys., Vol. 2, pp. 12 26, 1967.

11) Marshall, G., and E. Van Spiegel; On the Numerical Treatment of the Navier-Stokes Equations for an Incompressible Fluid, J. Engng. Math., Vol. 7, No. 2, pp. 173 188, 1973.

12) Temam, R.: Sur l'Approximation delà Solution des Équations de Navier-Stokes par la Methode des Pas Fractionnaires (I). Arch. Rati. Mech. Anal., Vol. 32, pp. 135 153, 1969.

13) Temam, R.: Navier-Stokes Equations, Studies in Mathematics and Its Applications, eds. by J. L. Lions et al., North Holland, 1977.
14) Hirt, C. W., and B. D. Nichols; Adding Limited Compressibility to Incompressible Hydrocodes, J. Computational Phys., Vol. 34, pp. 390 400, 1980.

15) Kawahara, M.: Convergence of Finite Element Lax-Wendroff Method for Linear Hyperbolic Differential Equation, Proc. J.S.C.E., No. 253, pp. 95 107, 1976.

16) Kawahara, M., N. Takeuchi and T. Yoshida: Two Step Explicit Finite Element Method for Tsunami Wave Propagation Analysis, Int. J. Num. Meth. Engng., Vol. 12, pp. 331 351, 1978.

17) Kawahara, M.: Steady and Unsteady Finite Element Analysis of Incompressible Viscous Fluid, Finite Elements in Fluids, Vol. 3, (eds. by R. H. Gallagher, O. C. Zienkiewicz, J. T. Oden, M. M. Cecchi and C. Taylor) pp. 26 54, John Wiley \& Sons, 1978.

18) Kawahara, M.: On Finite Element Methods in Shallow Water Long Wave Flow Analysis, Computational Methods in Non-linear Mechanics, (ed. by J. T. Oden), pp. 161 187, NorthHolland, 1980.

19) Kawahara, M., and T. Yokoyama: Finite Element Method for Direct Runoff Flow, Proc. A.S.C.E., Vol. 106, No. HY4, pp. 519 534, 1980.

20) Kawahara, M., S. Nakazawa, S. Ohmori and T. Takagi: Two-step Explicit Finite Element Method for Storm Surge Propagation Analysis, Int. J. Num. Meth. Engng., Vol. 15, pp. 1129 1148, 1980.

21) Kawahara, M. and K. Hasegawa: SEADASER-A General Purpose Finite Element Program for Coastal Sea Current and Dispersion, Advances in Engng. Software, Vol. 3, No. 1, pp. 2 8, 1980.

22) Kawahara, M., H. Hirano, K. Tsubota, and K. Inagaki: Selective Lumping Finite Element Method for Shallow Water Flow, Int. J. Num. Meth. Fluid., Vol. 2, pp. 89 112, 1982.

23) Ito, M. I. Okauchi and T. Miyata: Wind Resisted Structures, Maruzen, 1978 (In Japanese).

(Received July 16, 1982) 